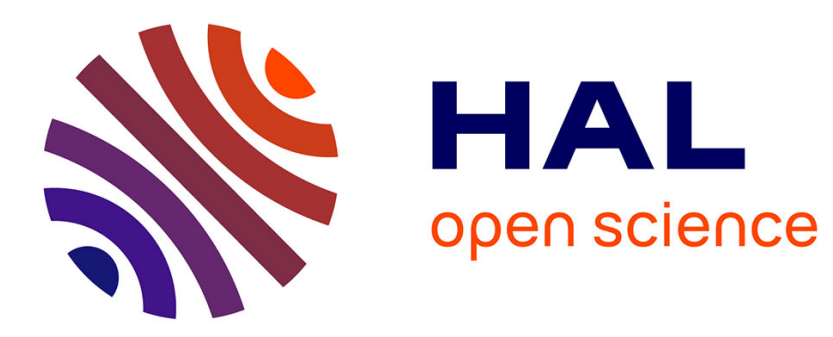

\title{
Optimal power flow in multi-terminal HVDC grids with offshore wind farms and storage devices
}

Miguel Jiménez Carrizosa,, Fernando Dorado Navas,, Gilney Damm, Françoise Lamnabhi-Lagarrigue

\section{- To cite this version:}

Miguel Jiménez Carrizosa, Fernando Dorado Navas,, Gilney Damm, Françoise Lamnabhi-Lagarrigue. Optimal power flow in multi-terminal HVDC grids with offshore wind farms and storage devices. International Journal of Electrical Power \& Energy Systems, 2015, 65, pp.291-298. 10.1016/j.ijepes.2014.10.016 . hal-01090326

\section{HAL Id: hal-01090326 \\ https://hal.science/hal-01090326}

Submitted on 13 Jul 2020

HAL is a multi-disciplinary open access archive for the deposit and dissemination of scientific research documents, whether they are published or not. The documents may come from teaching and research institutions in France or abroad, or from public or private research centers.
L'archive ouverte pluridisciplinaire HAL, est destinée au dépôt et à la diffusion de documents scientifiques de niveau recherche, publiés ou non, émanant des établissements d'enseignement et de recherche français ou étrangers, des laboratoires publics ou privés. 


\title{
Optimal power flow in multi-terminal HVDC grids with offshore wind farms and storage devices
}

\author{
Miguel Jiménez Carrizosa ${ }^{1}$, Fernando Dorado Navas ${ }^{2}$, Gilney Damm ${ }^{3}$ and \\ Françoise Lamnabhi-Lagarrigue ${ }^{4}$ \\ LSS, Supélec, France ${ }^{1,4}$ Universidad de Sevilla, Spain, ${ }^{2}$ Evry-Val d'Essonne \\ University, France ${ }^{3}$ \\ Miguel.Jimenez@Iss.supelec.fr, fdorado@cartuja.us.es, gilney.damm@ibisc.fr, \\ lamnabhi@Iss.supelec.fr
}

\begin{abstract}
This work presents a power flow strategy for multi-terminal HVDC grids. Energy is mainly generated via renewable energy sources and there are nodes in the network with the possibility to store energy. This energy is generated taking into account real weather conditions in order to make the best scheduling of the system in a realistic approach. An optimization scheme is proposed in which all these elements are included as well as real operation constraints. Distribution losses are minimized for the whole network. This gives as a result a control strategy being able to deal with the whole system and its inherent constraints giving the framework for a multi-objective optimization control.
\end{abstract}

Keywords: Multi-terminal HVDC, optimization losses, offshore wind farms, storage devices, model predictive control.

\section{Introduction}

The difficulty of satisfying the constant growth in worldwide energy demand is exacerbated by a number of well-known factors regarding the supply and use of conventional energy resources (oil, gas, etc.): the inconvenient geographical location of many conventional energy production sites vis-à-vis their centres of use, the continuous price inflation accompanying these resources and, of course, the fact that their use releases undesirable emissions into the atmosphere. These disadvantages are turning the world's attention towards a number of renewable energy solutions, among which offshore wind farms present a particular interest. There are several advantages to developing offshore wind energy, notably: wind conditions are more favorable out to sea than on land; the average wind speed is higher therefore providing more energy, and the wind direction tends to remain more constant in the absence of obstacles [1]. Furthermore, the wind is less turbulent because variations in temperature between the different layers of air are smaller over the sea than over land. For these reasons, offshore wind 
turbines have a larger up-time than onshore turbines. These are some of the reasons why wind farm investment is chiefly being channeled to offshore facilities rather than to their land-based equivalent despite their higher initial installation costs.

Energy transmission has historically been carried out in alternative current (AC). However high voltage direct current (HVDC) transmission is investigated in this study due to several advantages it possesses over AC lines. First of all the reactive power makes AC transmission losses greater than HVDC applications. Additionally, transmission capacity is also greater in HVDC lines due to the non-existence of the skin effect, and also the efficiency and controllability of DC converters are higher [2, 3, 4, 5]. Another advantage of using HVDC is that the use of fewer cables in DC lines also implies lower costs and weight enabling therefore the possibility of operating in remote marine regions where wind conditions are even more favorable [6, 7].

Although multi-terminal HVDC systems are technically feasible, they have not been widely accepted as a cost-effective transmission solution. There are only two HVDC installations which have operated as multi-terminal systems, but only for a limited time [8]. The first one is the HVDC Italy-Corsica-Sardinia [9] and the other one is the Quebec-New England Multi-terminal HVDC [10, 11]. These isolated applications highlight the fact that real exploitation of HVDC lines is still an open problem, involving technological and implementation issues.

In AC power systems, the optimal power flow (OPF) problem is defined by nonlinear, non-convex equations. In HVDC systems where there is no reactive power involved, the OPF problem is less complex but still retains its nonlinear characteristic when voltage control and optimal storage operation are included in the formulation. There are several different methods of solving the resulting nonlinear system of equations. Newton-Raphson (NR) is the most popular method in electrical engineering [12]. The solutions can be easily obtained through the equations' linearization. The main disadvantage is that neither the convergence of the method nor the achievement of the global optimum point are guaranteed. Furthermore, in the case of power systems a slack bus is usually considered when this method is applied. This fact entails risks for the proper operation of the system, such as the loss of the slack bus, that would cause the loss of the reference and consequently the abandon of the equilibrium because the method is not applicable. There are also other limitations to this method, which lie in the difficulty to handle the optimal management of energy storages and constraints on the capacity available in the transmission lines.

More recently, some authors have proposed other solutions to solve the problem. For example in [13] a multi-terminal DC power flow with a conventional AC power flow has been proposed. Or in [14] a steady-state multi-terminal DC model for power flow programs has been developed which allows to include converter limits as well as different converter topologies. However, both methods are based on the iterative resolution of the NR method until a solution is found within a tolerance value. Others authors have solved the problem applying new techniques as in [15] where the problem is solved by genetic algorithm that is an evolutionary-based heuristic algorithm.

This work presents a Power Flow formulation for HVDC networks in which transmission losses are minimized. The proposed optimization problem yields a solution where the voltage values in network nodes minimizes the transmission losses and will also account for all the constraints taken into consideration as it will be presented in 
section 2. This formulation results in a quadratic convex objective function but with non convex constraints. The existence of the solution for this problem is also discussed in sections 2.4 and 2.6 .

Other authors like [16], proposed an optimal power flow in order to minimize the losses in a multi-terminal HVDC grid. They considered the network state at each sample time for which they formulated an optimization problem regarding the transmission losses. However, in practical applications of variable energy power sources, it is interesting considering storage devices in order to improve the stability and the efficiency of the whole system [17]. In [18] an economic and environmental dispatch problem for a smart grid composed of multiple sources of generation systems (both conventional and renewable), consuming nodes and storage systems are proposed using the energy hub formulation. Their formulation, including weather forecasts to optimally operate the storage systems, was stated under the model predictive control approach, MPC, resulting in a mixed integer quadratic optimization problem.

The control strategy presented in this work takes into account the presence of storage devices in the HVDC networks. Their scheduling will depend on the power demand the network must satisfy and the weather forecast which is directly correlated to the power generation in the system.

\subsection{HVDC network}

We consider a general multi-terminal HVDC network connecting wind farms, storage devices and AC grids (see figure 1). As the wind is an intermittent energy source, not only does it provide the primary energy to be transformed into electrical form but it also constitutes a source of disturbances both in terms of quality of the energy produced and in the overall stability of the system. Consequently and as we mentioned above, storage devices will be placed strategically in order to cope with possible peaks of energy demand or low energy availability in the production nodes.

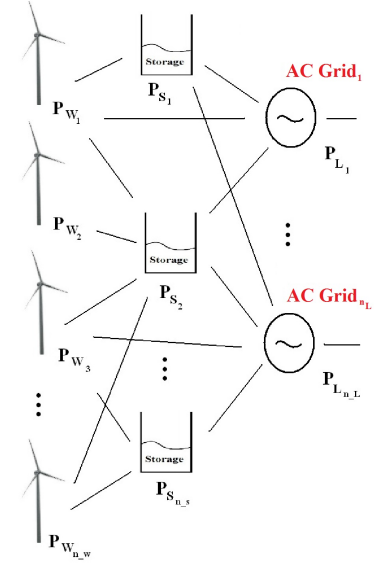

Figure 1: Multi-terminal HVDC Grid.

We will consider a scenario in which the AC networks connected to the grid will demand variable power, either because the load is variable or because market negotia- 
tion leads to that operating point. Wind farms together with the storage devices must be able to supply the consumed power via an HVDC grid. To tackle this problem, our work has used real wind measures [19] and we have considered weather forecasting [20, 21]. In this way the control scheme only has to deal with the prediction error instead of dealing with the full amount of variability.

Finally, as the sample time used is in the order of minutes, we can neglect the dynamics of cables, and we can use resistive models.

This document is organized as follows: in section 2 the optimization problem for the power flow within the HVDC network, as well as the constraints considered, is formulated. Also the existence of solutions for the proposed problem is discussed. Afterwards an application example is presented in section 3 , in the form of a practical implementation for a six-terminal case and, finally, in section 4 the main conclusions are drawn.

\section{Methodology}

\subsection{Nomenclature}

$Y \equiv$ Admittance matrix.

$N_{p} \equiv$ Prediction horizon

$P_{i}^{k} \equiv$ Active power at instant $k$ in node $i$.

$u_{i}^{k} \equiv$ Voltage at instant $k$ in node $i$.

$\left[u^{k}\right] \equiv$ Vector of voltages at instant $k . \quad T \equiv$ Sample time.

\subsection{Formulation of the problem}

The admittance matrix of an electrical grid, either DC or AC, has intrinsically all the information about its topology. This matrix is symmetric and has the form:

$$
[Y]=\left[\begin{array}{ccccc}
Y_{1,1} & \cdots & -Y_{1, i} & \cdots & -Y_{1, n} \\
\vdots & \ddots & \vdots & & \vdots \\
-Y_{1, i} & \cdots & Y_{i, i} & \cdots & -Y_{n, i} \\
\vdots & & \vdots & \ddots & \vdots \\
-Y_{1, n} & \cdots & -Y_{n, i} & \cdots & Y_{n, n}
\end{array}\right]
$$

This matrix shown in equation 1 has some interesting properties. It is always, at least, semi-positive defined. This is a consequence of one of its properties, that is, the sum of the elements of a row (or column because $Y$ is symmetric) is zero if there are not grounded resistances in this node, in which case the sum is equal to that element. Or in other words, if there are no elements connected to the ground at any node, the matrix is semi-positive definite, and it will have an eigenvalue equal to zero because the rows (or columns) are a linear combination of each other (the sum is zero).

Moreover, in DC grids, the active power in the node $i$ has the form:

$$
P_{i}=u_{i} \sum_{j=1}^{n} Y_{i, j} \cdot u_{j}
$$

If we see the whole DC grid in terms of power, there will be nodes that inject power into the system, there will be nodes that absorb power from the system and there 
will be nodes that neither supply nor absorb power, these last nodes are the internal interconnection nodes (they are inside the grid). The algebraic sum of all these powers must be equal to the losses in the system (transmission losses in the lines). Using this idea we can define a losses function, $F_{\text {Losses }}$, which depends on voltages of each node.

$$
F_{\text {Losses }}\left(u_{1}, \cdots, u_{n}\right)=\sum_{k=1}^{n} P_{i}=[u]^{t} \cdot[Y] \cdot[u]
$$

where $[u]=\left[u_{1}, \cdots, u_{n}\right] \in \mathbb{R}^{1 \times n}$, and $u_{i}$ is the voltage in node $i$.

Our purpose is to minimize the transmission losses in the system, or equivalently we want to find a set of voltage values which minimize our function shown in equation (3). Obviously, not any set of voltages will be valid, because several constraints must be guaranteed. However, DC systems present other advantages compared to AC, where a significant deviation with respect to the nominal state produces irreparable damage to rotative elements, because a deviation of the nominal voltage and/or power causes a variation in the frequency (or vice versa), which is critical for the proper operation of the system. In DC systems, due to the absence of frequency, the nominal voltage will not have as severe restrictions as in the AC systems, so we can talk of a "nominal strip" instead of a nominal value. In this zone, any value for the voltage guarantees the proper functioning of the system. It is in this context where we can find the optimal set for all voltages, which minimizes the losses.

The "nominal strip" will have to be subject to numerous restrictions (they will be explained below), and for this reason, the use of MPC is particularly interesting, since it is able to manipulate effectively systems with constraints [22].

In addition, it is possible to carry out the minimization through a longer horizon if we possess reliable wind and consumption forecasts since the MPC handles in a natural way these types of problems with prediction horizon, $N_{p}$. Fortunately this is possible because current forecasts provide a very high reliability [20, 21]. So we can carry out an optimization along $N_{p}$, and the result will be improved compared to making it at each instant in a single state way.

Furthermore, as renewable (variable) energies are used in this work, it is interesting to include in the grid storage devices, to store energy when there is an excess and to supply energy when there exits a shortage in the system. Also, and thanks to the use of MPC, it is possible to include the dynamics of these storages, with their efficiencies $\mu_{i}$, which, in general, will be different when they are charging or discharging. In equation (4), $E_{s}^{k}$ represents the energy at instant $k$ in the storage $s$, and $T$ is the sample time (we will consider that all sampling periods have the same duration).

$$
E_{s}^{k+1}=E_{s}^{k}-T \cdot \mu_{i} \cdot P_{s} \quad \forall s \in S
$$

On the other hand, in our overall system, there will exist four types of nodes, the production nodes, $W$, (in this case wind farms), consumption nodes, $L$, storage nodes, $S$, and internal interconnection nodes, $I C$. It must be stressed that in $I C$ nodes there is no power production or consumption. Our objective function will minimize the subject to a number of constraints over a prediction horizon $N_{p}$. These restrictions are:

- The production and load power must be equal to forecast values (6a). 
- The voltages at the nodes must be only in the "nominal strip" described above (6).

- The current lines can not exceed certain values (maximum admissible current through the cables), (6c).

- The storage devices can absorb or supply power until a maximum value (6d).

- Also we have taken into account that a storage can not provide (absorb) power if it is empty (full) (6).

- The balance of power has to be complied at all moments (6).

With all this, our control variables will be the power supplied or absorbed by storage devices. However, as the restrictions of the system are formulated in terms of voltage our problem consists in finding the best combination of voltages which minimize $F_{\text {Losses }}$. These founded values will be the references to a lower control.

With the aforementioned considerations and taking the transmission losses equation as described in (3) the optimization problem can be formulated as follows:

$$
\min \left(F_{\text {Losses }}\left(u_{1}^{k}, \cdots, u_{n}^{k+N_{p}}\right)\right)=\min \left(\sum_{k=1}^{N_{p}}\left[\mathbf{u}^{k}\right]^{t} \cdot[Y] \cdot\left[\mathbf{u}^{k}\right]\right)
$$

subject to:

a) $j \in W \cup L / \quad P_{j}=\left[\mathbf{u}^{k}\right]^{t} \cdot[Y]_{j}^{*} \cdot\left[\mathbf{u}^{k}\right] \quad \forall j$, and $\forall k$

b) $u_{i, \text { min }}^{k} \leq u_{i}^{k} \leq u_{i, \text { max }}^{k} \forall i$, and $\forall k$

$$
\text { c) }\left|\frac{u_{i}^{k}-u_{j}^{k}}{R_{i, j}}\right| \leq I_{\text {max }, l i n e_{i j}} \forall i, j, k
$$

$$
\text { d) } P_{s, \min } \leq P_{s}^{k} \leq P_{s, \max } \forall s \in S \text { and } \forall k
$$

e) $[E]_{s, \text { min }}^{k} \leq[E]_{s}^{k} \leq[E]_{s, \text { max }}^{k} \forall s \in S$ and $\forall k$

$$
\text { f) } \sum P_{W_{i}}^{k}-\sum P_{L_{i}}^{k}=\sum P_{S_{i}}^{k}-\sum P_{\text {Loss }}^{k} \forall k
$$

where $\left[\mathbf{u}^{k}\right]=\left[u_{1}^{k}, \cdots, u_{n}^{k}\right] \in \mathbb{R}^{1 \times n}$, and $u_{i}^{k}$ is the voltage in node $\mathrm{i}$ at instant $k$.

$$
[Y]_{j}^{*}=\left[\begin{array}{ccccc}
0 & \cdots & \frac{Y_{1, j}}{2} & \cdots & 0 \\
\vdots & \ddots & \vdots & & \vdots \\
\frac{Y_{1, j}}{2} & \cdots & Y_{j, j} & \cdots & \frac{Y_{n, j}}{2} \\
\vdots & & \vdots & \ddots & \vdots \\
0 & \cdots & \frac{Y_{n, j}}{2} & \cdots & 0
\end{array}\right] \in \mathbb{R}^{n \times n}
$$




\subsection{Advantages}

One of the biggest advantages of this method is that the losses are minimized with respect to a "classic" power flow, and this is particularly important since we increase the number of nodes. Other benefits must be pointed out, and one of the most significant is that there does not exist a balance node, so there is no dependence on such nodes, something crucial in methods that use node balance. Other advantage that can be obtained, is that, thanks to the implantation of the MPC, we can anticipate problems in the grid such as: over-voltages, over-currents, emptying or filling of storage devices, etc...

Also the storage models used are closer to reality as they include performance in charge and discharge (in general different).

\subsection{Existence of solutions}

The first question that we should ask is whether the proposed problem has a solution, and if there is solution we should also ask whether it is unique. In this section we will expose the demonstration that there are many possible solutions, but there is only one that gives the minimum value for $F_{\text {Losses }}$. For simplicity, firstly we carry out for the case of $\mathbb{R}^{3}$ ( 3 nodes) and then we will generalize to the case of $\mathbb{R}^{n}$ (n nodes).

\subsection{Existence of solutions in $\mathbb{R}^{3}$}

Our problem is to minimize a function subject to some restrictions. We are going to analyze the properties of these constraints to ensure that there will be a region in the space that fulfills all the constraints. In this explanation we will assume that $N_{p}=1$. This fact does not diminish the generality of the proof, it is only to make the calculations clearer.

\subsubsection{Constraints type a}

According to our defined problem, the first constraints are the type $j \in W \cup L$ / $P_{j}=[\mathbf{u}]^{t} \cdot[Y]_{j}^{*} \cdot[\mathbf{u}]$, and if we assume that we know the power in nodes 1 and $3:$

$$
\begin{aligned}
& {\left[\begin{array}{lll}
u_{1} & u_{2} & u_{3}
\end{array}\right] \cdot\left[\begin{array}{ccc}
Y_{1,1} & -\frac{Y_{1,2}}{2} & -\frac{Y_{1,3}}{2} \\
-\frac{Y_{1,2}}{Y_{1,3}} & 0 & 0 \\
-\frac{Y_{1,3}}{2} & 0 & 0
\end{array}\right] \cdot\left[\begin{array}{l}
u_{1} \\
u_{2} \\
u_{3}
\end{array}\right]=P_{1}} \\
& {\left[\begin{array}{lll}
u_{1} & u_{2} & u_{3}
\end{array}\right] \cdot\left[\begin{array}{ccc}
0 & 0 & -\frac{Y_{1,3}}{2} \\
0 & 0 & -\frac{Y_{2,3}}{2} \\
-\frac{Y_{1,3}}{2} & -\frac{Y_{2,3}}{2} & Y_{3,3}
\end{array}\right] \cdot\left[\begin{array}{l}
u_{1} \\
u_{2} \\
u_{3}
\end{array}\right]=P_{3}}
\end{aligned}
$$

These restrictions are clearly shaped in a quadric. We are going to analyze the quadric of equation (7), noting that the development is analogous to the other equation (8).

Firstly we want to know the nature of its center $\mathbf{c}=\left[x_{0}, y_{0}, z_{0}\right]$.

$$
\left[\begin{array}{ccc}
Y_{1,1} & -\frac{Y_{1,2}}{2} & -\frac{Y_{1,3}}{2} \\
-\frac{Y_{1,2}}{Y_{1,3}^{2}} & 0 & 0 \\
-\frac{Y_{2}}{2} & 0 & 0
\end{array}\right] \cdot\left[\begin{array}{l}
x_{0} \\
y_{0} \\
z_{0}
\end{array}\right]=0 \Rightarrow \mathbf{c}=\left[\begin{array}{c}
0 \\
-\frac{Y_{1,3}}{Y_{1,2}} \cdot a \\
a
\end{array}\right]
$$


As the system of equation (9) is an under-determinate compatible system, there are infinite centers that are on the line $\mathbf{r} \equiv u_{2}=-\frac{Y_{1,3}}{Y_{1,2}} \cdot u_{3}$ (in the plane $u_{1}=0$ ).

Since matrix $Y$ is symmetric, by the theorem of Schur there exists an orthogonal matrix $P$ such that $D=P^{t} Y^{*} P$, where $D=\operatorname{diag}\left(\lambda_{1}, \lambda_{2}, \lambda_{3}\right)$ and $\lambda_{i} \forall i=1,2,3$ are the eigenvalues of $Y^{*}$.

The eigenvalues of $Y^{*}$ has the form:

$$
\left|\lambda \cdot I-Y^{*}\right|=0 \Longleftrightarrow\left\{\begin{array}{c}
\lambda_{1}=0 \\
\lambda_{2}=\frac{Y_{1,1}+\sqrt{Y_{1,1}^{2}+Y_{1,2}^{2}+Y_{1,3}^{2}}}{2} \triangleq \frac{\theta_{2}}{2} \\
\lambda_{3}=\frac{Y_{1,1}-\sqrt{Y_{1,1}^{2}+Y_{1,2}^{2}+Y_{1,3}^{2}}}{2} \triangleq \frac{\theta_{3}}{2}
\end{array}\right.
$$

By definition of the admittance matrix $Y$, it is always true that $Y_{i, i} \geq \sum_{j=1, j \neq i}^{N} Y_{i, j}$, by other way it is also true that: $\sqrt{Y_{1,1}^{2}+Y_{1,2}^{2}+Y_{1,3}^{2}}>Y_{1,1}$, and for this reason $\lambda_{2}>0$ and $\lambda_{3}<0$. So, matrix $Y^{*}$ has zero, positive and negative eigenvalues. This fact makes power constraints of this kind non-convex restrictions.

An orthonormal base, $\mathfrak{B}_{1}$, made up by eigenvectors associated to each eigenvalue is $\mathfrak{B}_{1}=\left\{\overrightarrow{v_{1}}\left|\overrightarrow{v_{2}}\right| \overrightarrow{v_{3}}\right\}$, where:

$$
\begin{gathered}
\overrightarrow{v_{1}}=\frac{1}{\sqrt{Y_{1,2}^{2}+Y_{1,3}^{2}}}\left[\begin{array}{c}
0 \\
-Y_{1,3} \\
Y_{1,2}
\end{array}\right], \overrightarrow{v_{2}}=\frac{1}{\sqrt{\theta_{2}^{2}+Y_{1,2}^{2}+Y_{1,3}^{2}}}\left[\begin{array}{c}
\theta_{2} \\
-Y_{1,2} \\
Y_{1,3}
\end{array}\right] \\
\overrightarrow{v_{3}}=\frac{1}{\sqrt{\theta_{3}^{2}+Y_{1,2}^{2}+Y_{1,3}^{2}}}\left[\begin{array}{c}
-\theta_{3} \\
Y_{1,2} \\
Y_{1,2}
\end{array}\right]
\end{gathered}
$$

Thus making a change of variables of the form:

$$
\left[\begin{array}{l}
u_{1} \\
u_{2} \\
u_{3}
\end{array}\right]=[P]\left[\begin{array}{l}
x_{1} \\
y_{1} \\
z_{1}
\end{array}\right]+\mathbf{c}
$$

we obtain the reduced form of quadric $S_{1}$ shown in (7):

$$
\begin{gathered}
S_{1} \equiv \alpha y_{1}^{2}+\beta z_{1}^{2}=P_{1} \\
\alpha=\frac{Y_{1,1} \theta_{2}^{2}+Y_{1,2}^{2} \theta_{2}+Y_{1,3} \theta_{2}^{2}}{\theta_{2}^{2}+Y_{1,2}^{2}+Y_{1,3}^{2}}>0 \\
\beta=\frac{Y_{1,1} \theta_{3}^{2}+Y_{1,2}^{2} \theta_{3}+Y_{1,3} \theta_{3}^{2}}{\theta_{3}^{2}+Y_{1,2}^{2}+Y_{1,3}^{2}}<0
\end{gathered}
$$

also if we simplify the operations, we obtain that $\alpha=\lambda_{2}$ and $\beta=\lambda_{3}$ so:

$$
S_{1} \equiv \lambda_{2} y_{1}^{2}+\lambda_{3} z_{1}^{2}=P_{1}
$$

therefore the quadric is a hyperbolic cylinder as figure 2 shows. 


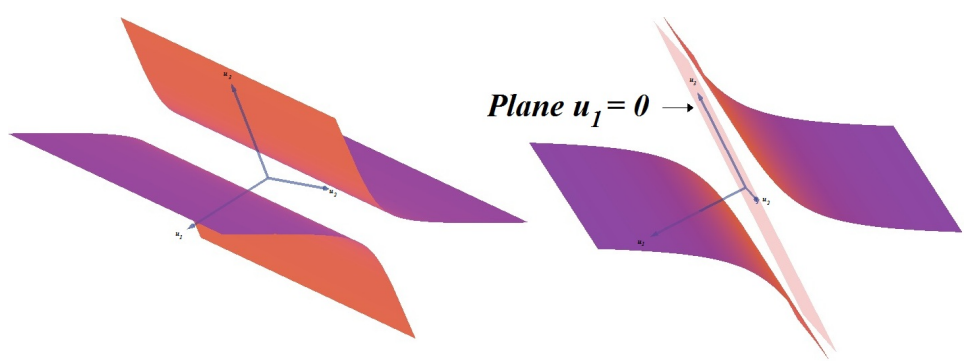

Figure 2: Hyperbolic cylinder $S_{1}$.

If we proceed in an analogous manner to the case of the load, we obtain the reduced form of the quadric $S_{3}$ shown in (8):

$$
S_{3} \equiv \lambda_{1}^{\prime} x_{3}^{2}+\lambda_{2}^{\prime} y_{3}^{2}=P_{3}
$$

where $\lambda_{1}^{\prime}$ and $\lambda_{2}^{\prime}$ are the eigenvalues of the matrix of system (8). It can now be noted, that the hyperbolic cylinder is symmetric with respect to the plane $u_{3}=0$, and also the axes $x_{3}, y_{3}, z_{3}$ are different to $x_{1}, y_{1}, z_{1}$.

The intersection of two quadric, figure 3 , gives a curve, $\mathfrak{C}$, which can be parametrized, and it contains all the values of $u_{1}, u_{2}$ and $u_{3}$ in that our function objective must be minimized. $\mathfrak{C}$ can be defined as:

$$
\mathfrak{C} \equiv\left\{\left(u_{1}, u_{2}, u_{3}\right) \in \mathbb{R} s / S_{1} \cap S_{3}\right\}
$$

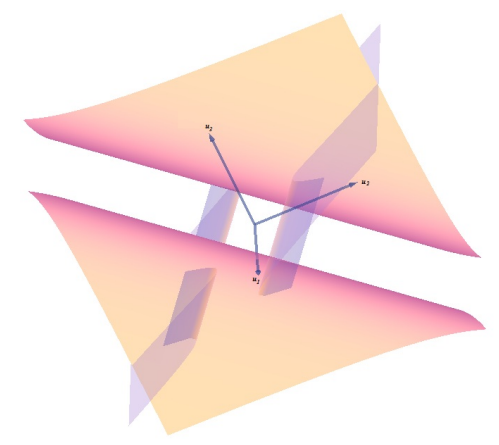

Figure 3: Intersection of hyperbolic cylinders.

\subsubsection{Constraints type $b$}

The second type of restrictions is related to the "nominal strip" values for the voltages at each node. Graphically, these constraints form a cuboid whose side lengths are the lengths of the intervals for each voltage at each node. 


\subsubsection{Constraints type c}

This type of constraints is related to the maximum transmission capacity of the lines (maximum current through the lines). They are of the type $\left|\frac{u_{i}-u_{j}}{R_{i, j}}\right| \leq I_{\text {maxline }}$. In $\mathbb{R}^{3}$ these restrictions are easy to plot, they have the form shown in figure 4 , and the set of correct values is the space within the intersection of all these planes (and remember that only the octant of the space where the variables are positive has a physical interpretation).

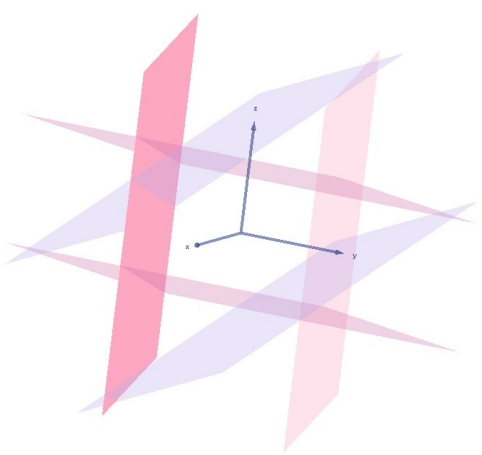

Figure 4: Current lines constraints.

\subsubsection{Other constraints}

The other kinds of restrictions (type $d, e$ and $f$ ) are not geometrically significant, because they have no a direct relation with the voltage values.

\subsubsection{Region of solutions in this case}

The curve $\mathfrak{C}$, given by equation $(16)$, is always within the bounds stated by constraints 2.5 .2 and 2.5.3. Besides, in all cases, the curve $\mathfrak{C}$ will exist because it is by definition the intersection between two hyperbolic cylinders, and these quadrics are each symmetric with respect to a perpendicular plane (in this case $u_{1}=0$ and $u_{3}=0$ ). So, in general, the curve $\mathfrak{C}$ is in a non null region in $\mathbb{R}^{3}$ where the optimum point lies.

\subsection{Existence of solutions in $\mathbb{R}^{n}$}

If we proceed in analogous form of $\mathbb{R}^{3}$ we can achieve a set of values in $\mathbb{R}^{n}$ where all restrictions are satisfied.

\subsubsection{Constraints type a}

Now, we assume that there are $n=n_{w}+n_{S}+n_{L}$ nodes, where $n_{w}$ is the number of production nodes, and they form a subspace with dimension $n_{w}$, and something similar 
for $n_{L}$ (number of load nodes). Once again, we have $j \in W \cup L / P_{j}=[u]^{t} \cdot[Y]_{j}^{*} \cdot[u]$, where:

$$
[Y]_{j}^{*}=\left[\begin{array}{ccccc}
0 & \cdots & \frac{Y_{1, j}}{2} & \cdots & 0 \\
\vdots & \vdots & \vdots & \vdots & \vdots \\
\frac{Y_{1, j}}{2} & \cdots & Y_{j, j} & \cdots & \frac{Y_{n, j}}{2} \\
\vdots & \vdots & \vdots & \vdots & \vdots \\
0 & \cdots & \frac{Y_{n, j}}{2} & \cdots & 0
\end{array}\right]
$$

It is again a quadric in non reduced form, and therefore we can obtain a change of variable to obtain the reduced form of the quadric. If we calculate the eigenvalues of these matrices, they are:

$$
\left|\lambda \cdot I-Y^{*}\right|=0 \Longleftrightarrow\left\{\begin{array}{c}
\lambda_{1}=0 \\
\vdots \\
\lambda_{n-2}=0 \\
\lambda_{n-1}=\frac{Y_{i, i}+\sqrt{\sum_{k=1}^{n} Y_{i, k}^{2}}}{2} \\
\lambda_{n}=\frac{Y_{i, i}-\sqrt{\sum_{k=1}^{n} Y_{i, k}^{2}}}{2}
\end{array}\right.
$$

and as $Y_{i, i}<\sqrt{\sum_{k=1}^{n} Y_{i, k}^{2}}$ is always true, we obtain for all the cases that these matrices $[Y]^{*}$ have one positive eigenvalue, one negative eigenvalues and the rest of them are zero. So the quadrics have the form of hyperbolic cylinders in the space $\mathbb{R}^{n}$, which also are intersected because each of them is centered in the corresponding plane whose variable is equal to zero $u_{i}=0$. That is, they are perpendicular to each other.

\subsubsection{Constraints type $b$}

In $\mathbb{R}^{n}$, these restrictions form hypercubes bounding maximum and minimum values of voltages.

\subsubsection{Constraints type $c$}

In the case of $\mathbb{R}^{n}$, the restrictions about the maximum transmission capacity are planes of order 2 , and the intersection of all of them form a region of space $\mathbb{R}^{n}$. In this case we only work in the region of space where all the variables are positive.

\section{Application case: a six-terminal system}

The simulations for the proposed method will be carried out in the system shown in Figure 5, where there are two wind farms, two storage systems and two AC networks, in which there are different consumptions. Note also, that geographically and by proximity, the whole system could be divided into two subsystems, each one with a wind farm, a load and a storage device. This does not mean that they work separately, but if it is true that from the energetic point of view, it will be more reasonable to supply power 


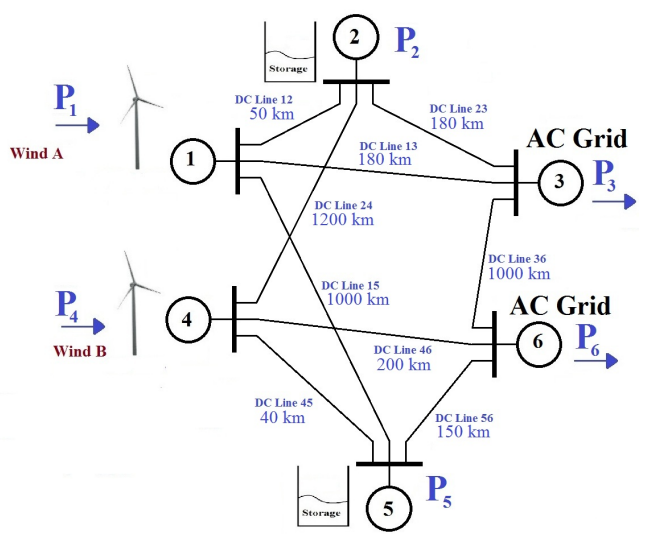

Figure 5: Six-terminal simulation grid.

from node 1 to nodes of its subsystem, rather than to the other subsystem. Although, there will be cases, included in the simulations, where this does not happen.

Observing the model shown in equation (5), we have to minimize a nonlinear function subject to both linear and nonlinear constraints. To solve this problem we have used a tool called Opti Toolbox and simulations have been carried out in the MATLAB (c) environment.

Table 1 lists the parameter values and constraints used for the model of the whole system. We have supposed that when we are at instant $k$, we have a forecast for the next 2 hours. And, as our sampling time is $15 \mathrm{~min}$, then $N_{p}=8$.

According to table 1. we have considered that the "nominal voltage strip" is $\pm 10 \%$ with respect to $100 \mathrm{kV}$. Also, we have taken into account that the efficiency of each storage is different, and the nominal power too. It shows how all possible cases are easily executable thanks the formulation of the problem. With respect to HVDC transmission cables, we have chosen typical values for them. As explained above, we will only consider resistive models, due to the selected sample time, thereby ignoring the dynamics of cables.

With these data and constraints we have carried out simulations for a typical week. We have used real measures of wind [19] as we observe in figure 6 .

In figure 7, real demand predictions are shown for a week, for two different areas [23]. We have selected the criterion that a negative power means that the concern node absorbs energy from the system.

With these two premises and starting from initial conditions in the energy levels of the storage devices, as shown in table 1. we operate the system with a view to minimizing losses with a prediction sliding horizon of $2 \mathrm{~h}$. The results that we have obtained are as follows.

If we look closely at figures 6,7 and 8 , we can point out many interesting moments which occurred throughout the week.

On the first day, in the subsystem 1 there was more wind than consumption, so the storage in node 2 was charged. With respect to subsystem 2 , there was less wind than 
Table 1: Simulation parameter values.

\begin{tabular}{|c|c|c|}
\hline Prediction Horizon & $N_{p}$ & 2 hours \\
\hline Sampling Time & $T$ & $15 \mathrm{~min}$ \\
\hline Nominal power of wind farm 1 & $P_{n o m-w f-1}$ & $400 \mathrm{MW}$ \\
\hline Nominal power of wind farm 2 & $P_{\text {nom }-w f-2}$ & $300 \mathrm{MW}$ \\
\hline Nominal voltage of DC grid & $u_{\text {nom }}$ & $100 \mathrm{kV}$ \\
\hline Nominal power of DC grid & $P_{\text {nom }}$ & $100 \mathrm{MW}$ \\
\hline Minumum voltage in all nodes & $u_{i, \min }$ & 0.9 p.u \\
\hline Maximum voltage in all nodes & $u_{i, \max }$ & $1.1 \mathrm{p} . \mathrm{u}$ \\
\hline Maximum current per line 12 & $i_{\max -l i n e} 12$ & 1 p.u. \\
\hline Maximum current per line 13 & $i_{\max -l i n e} 13$ & 1 p.u. \\
\hline Maximum current per line 15 & $i_{\max -\text { line } 15}$ & 1 p.u. \\
\hline Maximum current per line 23 & $i_{\max -\text { line } 23}$ & 1 p.u. \\
\hline Maximum current per line 24 & $i_{\max -\text { line } 24}$ & 1 p.u. \\
\hline Maximum current per line 36 & $i_{\max -\text { line } 36}$ & 1 p.u. \\
\hline Maximum current per line 45 & $i_{\max -l i n e} 45$ & 1 p.u. \\
\hline Maximum current per line 46 & $i_{\max -\text { line } 46}$ & 1 p.u. \\
\hline Maximum current per line 56 & $i_{\text {max-line } 56}$ & 1 p.u. \\
\hline Resitance cables & $R_{\text {cable }}$ & $0.121 \Omega / \mathrm{km}$ \\
\hline Length line 12 & & $50 \mathrm{~km}$ \\
\hline Length line 13 & & $180 \mathrm{~km}$ \\
\hline Length line 15 & & $1000 \mathrm{~km}$ \\
\hline Length line 23 & & $180 \mathrm{~km}$ \\
\hline Length line 24 & & $1200 \mathrm{~km}$ \\
\hline Length line 36 & & $1000 \mathrm{~km}$ \\
\hline Length line 45 & & $40 \mathrm{~km}$ \\
\hline Length line 46 & & $200 \mathrm{~km}$ \\
\hline \multirow[t]{2}{*}{ Length line 56} & & $150 \mathrm{~km}$ \\
\hline & Storage 1 & Storage 2 \\
\hline Energy at initial time & 3.6 GWh $(60 \%)$ & 1.5 GWh $(50 \%)$ \\
\hline Maximun power charging & $350 \mathrm{MW}$ & $220 \mathrm{MW}$ \\
\hline Maximun power discharging & $300 \mathrm{MW}$ & $200 \mathrm{MW}$ \\
\hline Charge efficiency $\eta_{c 1}$ & 0.8 & \\
\hline Discharge efficiency $\eta_{d 1}$ & 0.8 & \\
\hline Charge efficiency $\eta_{c 2}$ & & 0.85 \\
\hline Discharge efficiency $\eta_{d 2}$ & & 0.85 \\
\hline
\end{tabular}




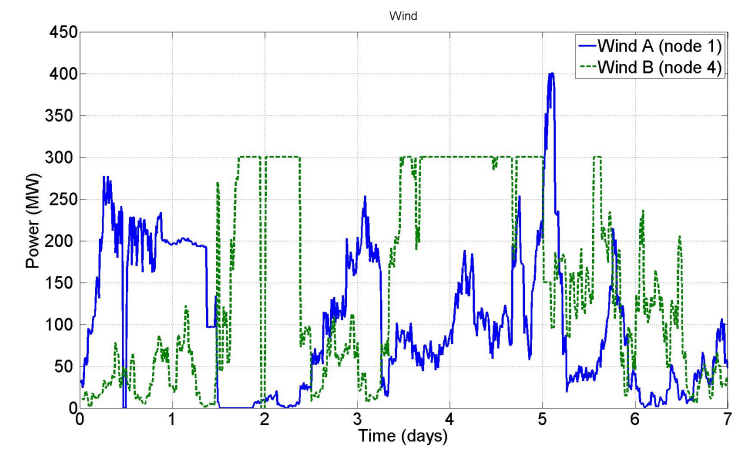

Figure 6: Wind power in production nodes for a typical week.

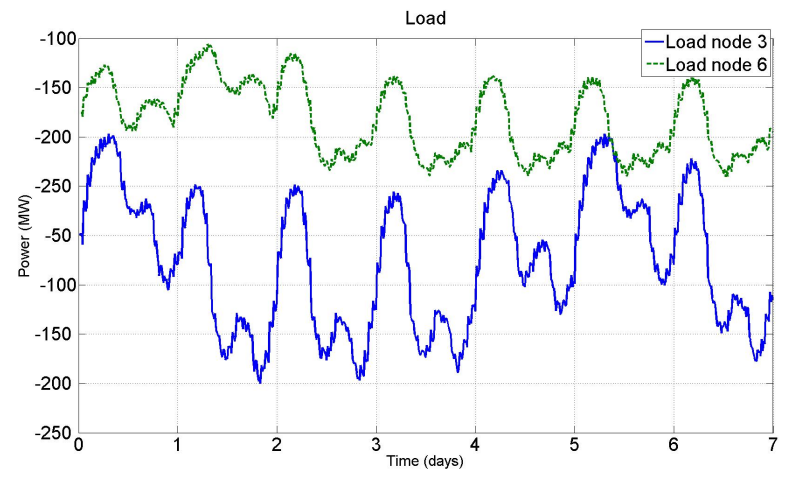

Figure 7: Consumption power for a typical week.

load so that the storage in node 5 was discharged. Between the end of the second day and the beginning of the third, it is clearly shown that the trend was in reverse, so the storage at node 5 was charged and the other was discharged. But perhaps the most interesting moment occurs when the storage at node 5 is filled (at the end of the fourth day), we observe that from this moment, although there was more wind than load in subsystem 2, the energy went to the storage device of the other subsystem, and this storage device is charging in spite of the fact that there was more consumption than generation in this subsystem. It is an unequivocal evidence that our control is working properly.

Another positive sign is that all constraints are satisfied. In effect, if we see figure 8 , we can confirm that any storage device was suppling (or consuming) power beyond their limits (see table 11). Something similar occurs if we observe figure $8 \mathrm{p}$, we can validate that when one storage is full, it does not absorb more power, and when it is empty, it does not supply power.

In figure 9 we see that all the voltages are always in the "nominal strip", so these restrictions are satisfied too. We can also point out that the voltages are nearer than 1.1 p.u, this is a fact that it should not surprise us, because to minimize losses it is clear 

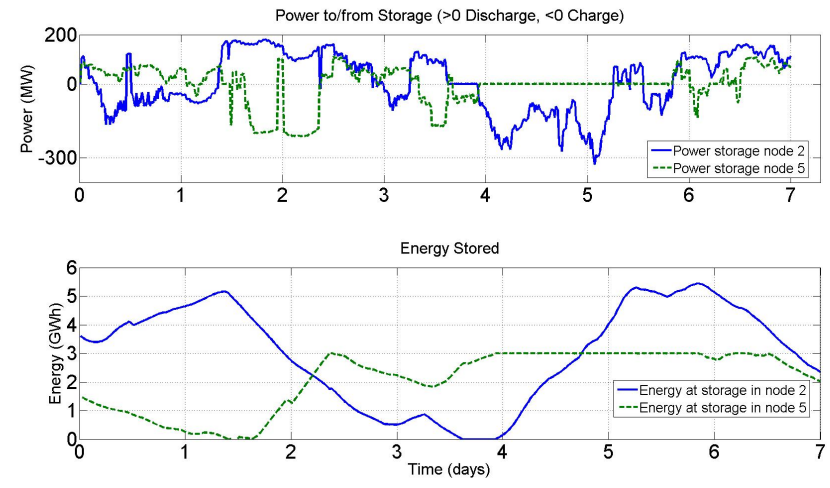

Figure 8: a) Power storage b) Energy storage.

that high voltage levels favor it. Moreover, it has to be stressed that when the storage at node 2 is full (on the fifth day) we can observe that the voltage regimes of subsystem 1 (the subsystem of this storage) are decreased, but they are always within the limits.

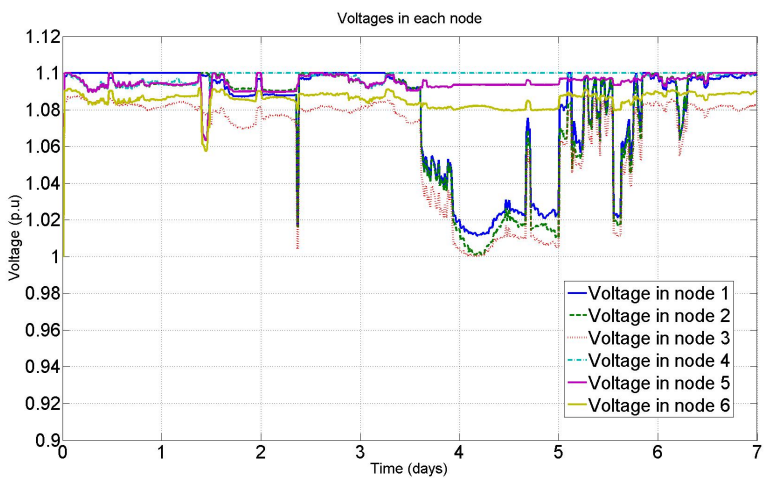

Figure 9: Voltages in each node.

In figure 10 we notice how all the currents through the lines do not exceed the maximum current that they can tolerate (the positive or negative values only indicate the power direction with respect to the reference).

\subsection{Losses comparative}

To appreciate more precisely the power of our optimal control, we are now going to compare the losses obtained with those that we would obtain if we carried out a classic NR method (explained in section 2) with the slack bus always in node 2. This comparison is shown in figure 11, and it is clear that with our proposed optimal controller the efficiency of the system is improved. In addition, if we compute the total losses in this week, we obtain that for the MPC the losses are 78.6 GWh and for the NR method they 


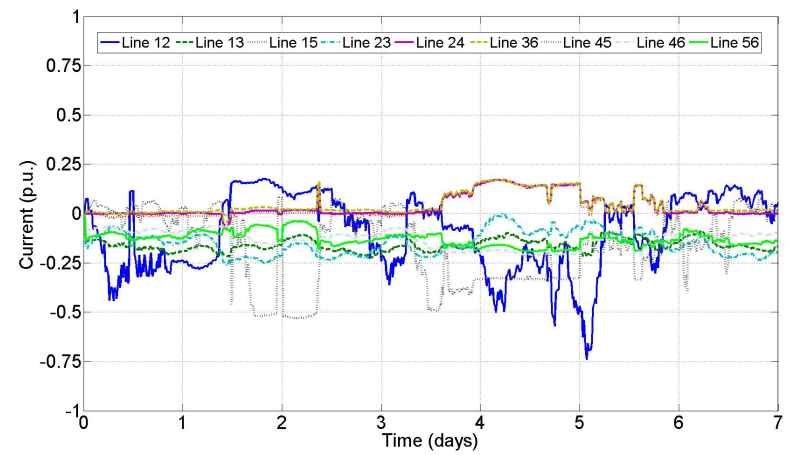

Figure 10: Currents thought the lines.

Table 2: Losses comparative.

\begin{tabular}{cc}
\multicolumn{2}{c}{ Total Losses } \\
\hline NR method & 194.3 GWh \\
MPC method & 78.6 GWh
\end{tabular}

are $194.3 \mathrm{GWh}$, that is, a $60 \%$ reduction in losses with the optimal control (see table 2).

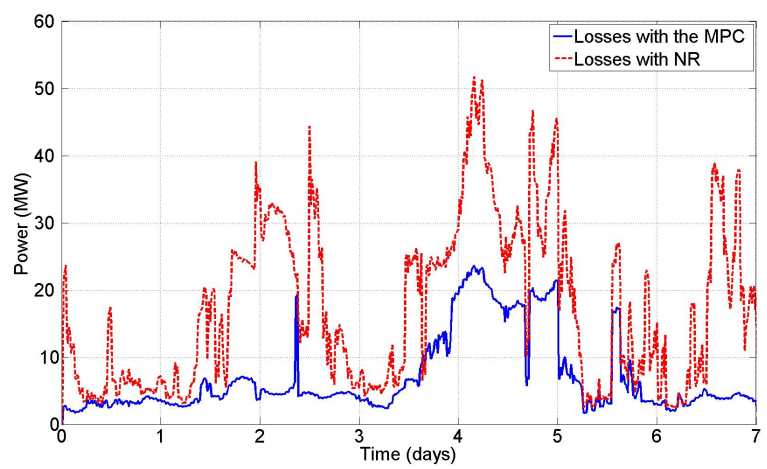

Figure 11: Losses comparison.

\section{Conclusions}

This paper has developed a control strategy, to be included in an hierarchical scheme, for a multi-terminal High Voltage Direct Current network. The control strategy includes weather forecasts and load predictions, and it optimizes the power flows in the network in order to: 
- minimize transmission losses,

- avoid power network congestions.

These goals are obtained by calculating the best set of voltages for each terminal, taking into account several restrictions (see section 2). A new formulation is proposed in this paper, which will help us to better handle the restrictions in order to optimize the problem. One of the key elements will be the storage devices, and they will allow us to manipulate the system optimally.

One of the drawbacks is that our control is sensitive to prediction/forecast errors, in particular concerning the wind speed. It is because the produced power from a wind turbine has a cubic dependence on the wind speed. Therefore, if there is a small difference between the forecast wind speed and the current speed, this may produce significant power errors. For this reason, it is necessary to rely on a lower level controller (not described in this paper) that must keep the system stable between sample times.

It is also noteworthy in the present work that the main criterion is to minimize the losses, but there may be moments that for security this is not the most advisable. Also, and thanks to the proposed formulation, it is possible to operate the system with secondary criterion such as maintaining the energy of the storage devices in an preestablished interval, or maintaining the voltages in a range more suitable for us.

\section{Further work}

One of the critical aspects of this report is the forecasts. Our controller works with a sample time in the order of minutes, the so-called very short space of time forecast (10-15 min). Recent studies show that it is possible to use Markov methods to carry out it [20, 21]. Differences between weather forecasts and load predictions that arise in the real implementation of this scheme are tackled through a lower controller of the system. Among them voltage margin control and droop control should be highlighted [24].

\section{Acknowledgements}

This work was supported by WINPOWER project (ANR-10-SEGI-016).

Fernando Dorado would like to acknowledge Seville University for funding this work under the "V Plan Propio de Investigación de la Universidad de Sevilla".

\section{References}

[1] EWEA, "Delivering Offshore Wind Power in Europe", European Wind Energy Association, 2007.

[2] J. Arrillaga, Y.H. Liu, N.R. Watson, "Flexible Power Transmission. The HVDC Options", Ed. John Wiley \& Sons, 2007.

[3] G. Asplund, "Ultra high voltaje trasmission”, ABB review, February 2007. 
[4] J. Kreuse, ”The future is now”, ABB review, March 2008.

[5] D. Ravemark and B. Normark, "Light and invisible”, ABB review, March 2005.

[6] A. Sannino, P. Sandeberg and L. Stendius, R.Görner, "Enabling the power of wind", ABB Review, March 2008 .

[7] R. Rudervall, J.P. Charpentier and R. Sharma, "High Voltage Direct Current (HVDC) Transmission Systems", Energy Week 2000, March 2000.

[8] D. Jovcic and B. T. Oo, ”Tapping on HVDC lines using DC transformers", Electric Power Systems Research, vol 81, no. 2, pp. 561-569, February 2011.

[9] V. Collet Billon, J.P. Taisne, V. Arcidiacono and F. Mazzoldi, ”The Corsican Tapping: from Design to Commissioning Tests of the Third Terminal of the Sardinia-Corsica-Ity HVDC Link", Power Delivery, IEEE transactions, vol. 4, no. 1, pp.794-799, January 1989.

[10] J.A Donahuea, D.A. Fisher, B.D. Raling and P.J. Tatroi, "Performance testing of the Sandy Pond HVDC converter", Power Delivery, IEEE transactions, vol. 8, no. 1, pp.422-428, January 1993.

[11] W.F. Long, J. Reeve, J.R. McNichol, M.S. Holland, J.P. Taisne, J. LeMay and D.J. Lorden, "Application aspects of multiterminal DC power transmission", Power Delivery, IEEE transactions, vol. 5, no. 4, pp.2084-2098, January 1993.

[12] P. Kundur, ” Power systems stability and contro"l, $1^{\text {st }}$ ed. Mc Graw Hill, 1993.

[13] W. Wang and M. Barnes, "Power Flow Algorithms for Multi-Terminal VSCHVDC With Droop Contro"l, Power Systems, IEEE Transactions on, vol 29, no. 4, pp. 1721-1730, July 2014.

[14] J. Beerten, S. Cole and R. Belmans, "Generalized steady-state VSC MTDC model for sequential AC/DC power flow algorithms", Power and Energy Society General Meeting (PES), 2013 IEEE, July 2013.

[15] U. Kiliç, K. Ayan and U. Arifoğlu, "Optimizing reactive power flow of HVDC systems using genetic algorithm", International Journal of Electrical Power \& Energy Systems, vol 55, pp. 1-12, February 2014.

[16] M. Aragés-Penalba, A. Egea-Àlvarez, O. Gomis-Bellmunt and A. Samper, "Optimum voltage control for loss minimization in HVDC multi-terminal transmission systems for large offshore wind farms", Electric Power Systems Research, vol 89, pp. 54-63, March 2012.

[17] A. Chakraborty, S. K. Musunuri, A. K. Srivastava and A. K. Kondabathini, ”Integrating STATCOM and Battery Energy Storage System for Power System Transient Stability: A Review and Applicatio"n, Advances in Power Electronics, 2012. 
[18] A.J. del Real, A. Arce and C.Bordons, "Combined environmental and economic dispatch of smart grids using distributed model predictive control", Electrical Power and Energy Systems, pp. 65-76, 2014.

[19] Goverment of UK, http://www.metoffice.gov.ukl

[20] R. Langella, A. Tesa and M. Giorgio, Very short-term probabilistic wind power forecasting based on Markov chain models, Probabilistic Methods Applied to Power Systems (PMAPS), 2010 IEEE 11th International Conference, pp. 107112. Singapore, June 2010.

[21] S.A. Pourmousavi-Kani and M.M. Ardehali, "Very short-term wind speed prediction: A new artificial neural networkMarkov chain model”, Energy Conversion and Management, vol. 52, pp. 738-745, August 2010.

[22] E.F. Camacho, C. Bordons, "Model Pedictive Control in the process industry”, Ed. Springer. 1995.

[23] OMIE, Operador del mercado ibérico de la energía, http: / / wWw . omie.es/ inicio/informacion-de-agentesl

[24] S. Shah, R. Hassan, and J. Suni, " HVDC transmission system architectures and control - A review", Control and Modeling for Power Electronics (COMPEL), 2013 IEEE 14th Workshop on, pp. 1-8, June 2013 